\title{
On the role of surface tensions and process conditions in detachment nanolithography
}

\author{
Jae Kwan Kim and Kahp Y. Suh ${ }^{\text {a) }}$ \\ School of Mechanical and Aerospace Engineering and the Institute of Advanced Machinery and Design, \\ Seoul National University, Seoul 151-742, Republic of Korea
}

(Received 31 March 2008; accepted 4 May 2008; published online 3 June 2008)

\begin{abstract}
We report on the role of intrinsic (adhesion force and wettability) and extrinsic (temperature and pressure) conditions to fabricate dense nanoscale patterns in detachment nanolithography. A phase diagram is constructed by using a rigiflex polymeric mold, an organic film, and silicon or gold substrate. Operating conditions in terms of surface tensions and processing parameters are discussed along with comparison of the minimum resolution with a simple theory. (C) 2008 American Institute of Physics. [DOI: 10.1063/1.2937143]
\end{abstract}

Detachment nanolithography is a reverse process of additive transfer methods ${ }^{1}$ and provides an alternative route to the fabrication of nanoscale patterns in a simple, one-step manner. A number of detachment-based patterning methods have been introduced with different mold (polymer, silicon) and film materials (metal, polymer, or organic layer). ${ }^{2-5} \mathrm{~A}$ key operating condition is a reasonable difference in adhesive forces between the mold/film interface $\left(W_{12}\right)$ and the film/substrate interface $\left(W_{23}\right)$, i.e., $W_{12}>W_{23}$. Despite extensive works in this field, the role of film wettability and process parameters as well as the interplay between them has not been well addressed. In this letter, we report on the effects of thermodynamic (adhesive forces and wettability) and process conditions (temperature and pressure) to realize dense nanoscale patterns in a typical experimental setup of detachment nanolithography.

For experiments, a nanoscale silicon master having $70 \mathrm{~nm}$ line-and-space patterns $(150 \mathrm{~nm}$ height, number of lines $=36$ per repeating unit) was used. The detailed geometry is shown in Fig. 1. A rigiflex polymeric mold of ultraviolet curable polyurethane acrylate (PUA) was replicated from this silicon master, which was used to detach nanoscale patterns from an underlying layer of $4,4^{\prime}$ bis $[N$-1-napthyl- $N$-phenyl-amino]biphenyl (NPB). Details on the synthesis and characterization of the PUA material were published elsewhere. ${ }^{6}$ Silicon (100) or gold-coated silicon wafer with a thickness of $100 \mathrm{~nm}$ using $\mathrm{Ti}(50 \mathrm{~nm})$ as an adhesion promoter (MHS1500A metal sputter, Moohan Co., Korea) was used as the substrate. For a detached layer, NPB was used instead of a polymer film because of its lower cohesion energy and stability in air. ${ }^{5}$ The thickness of NPB layer was $80-150 \mathrm{~nm}$, as measured by ellipsometry (L116B, Gaertner, USA) and rms roughness was $\sim 1.8 \mathrm{~nm}$. To achieve conformal contact between the mold and the substrate with uniform pressure distribution, a hydraulic pressure of $0.2-2$ bars was applied on the mold after inserting a thin polydimethyl siloxane (PDMS) slab as a buffer layer. While applying a pressure, temperature was increased to $90{ }^{\circ} \mathrm{C}$ for $20 \mathrm{~min}$ on a hot plate. The glass transition temperature $\left(T_{g}\right)$ of NPB is $\sim 96{ }^{\circ} \mathrm{C}$. Then, the sandwiched assembly of PDMS slab and PUA mold was disintegrated after

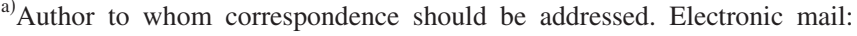
sky4u@snu.ac.kr. cooling to room temperature (cooling rate $\sim 2{ }^{\circ} \mathrm{C} / \mathrm{min}$ ) by using a sharp tweezer. The mold was fast removed from the substrate since a high detachment rate was reported to improve the physical removal of material from selected regions of a substrate. ${ }^{7}$ However, no significant difference in surface morphologies was observed for relatively slow detachment rates and different cooling rates. Detached patterns were examined by scanning electron microscopy (SEM) (S-48000, Hitachi Co., Japan) and atomic force microscopy (AFM) (XE-150, PSIA Co., Korea) measurements. Some AFM images were flatted but not further manipulated. A Ramé-Hart goniometer (Mountain Lakes) equipped with a video camera was used to measure static contact angles.

Figure 2(a) shows a phase diagram for four distinct surface morphologies (types I-IV) along with the corresponding SEM images, as dictated by the thermodynamic variables of spreading coefficient $(S)$ and work of adhesion ratio $\left(W_{12} / W_{23}\right)$. The demarcation lines are drawn from thermodynamic considerations and some mixed morphologies were observed near the vertical demarcation lines between types I and III and types II and IV. To construct this phase diagram, temperature and pressure were maintained constant at $90{ }^{\circ} \mathrm{C}$ and 1 bar, respectively. Also, surface energy and work of adhesion at the mold/film and the film/substrate interfaces were evaluated through the procedure reported earlier. ${ }^{8}$

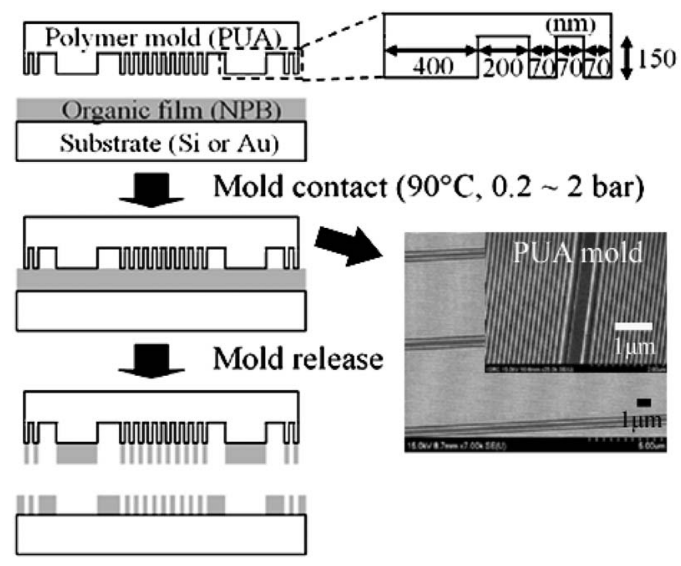

FIG. 1. A schematic procedure of the detachment nanolithography using PUA, NPB, Si, or Au as mold, film, and substrate, respectively. The geometry of the PUA mold is depicted in the figure. A SEM image of the replicated PUA mold is shown (lower right) with an enlarged view in the inset. 
(a)

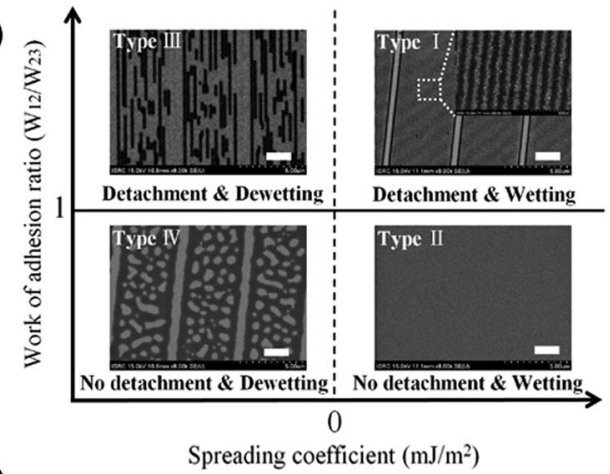

(b)

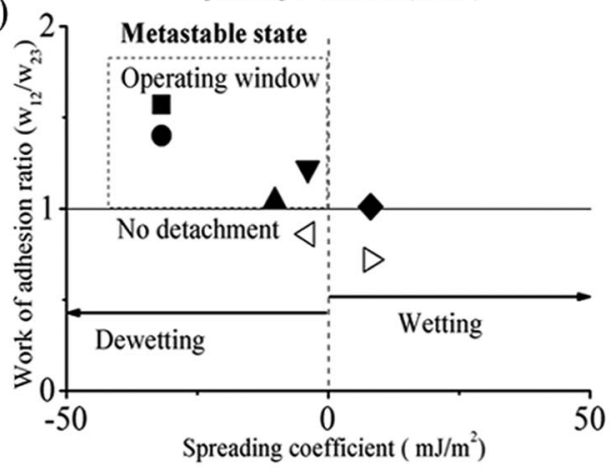

FIG. 2. (a) A phase diagram of detachment nanolithography with representative SEM images for four different regimes (Type I to IV), as dictated by the spreading coefficient and the work of adhesion ratio. The scale bar is $2 \mu \mathrm{m}$. (b) A phase diagram with different mold/film/substrate materials and process parameters (pressure, temperature) from the literature. Epoxy/Alq $/$ /ITO $\left(10\right.$ bar, $\left.80 \sim 120^{\circ} \mathrm{C}\right),{ }^{4}$ o: $\mathrm{PDMS} / \mathrm{Alq}_{3} / \mathrm{ITO}$ (0 bar, $\left.90^{\circ} \mathrm{C}\right){ }^{5} \boldsymbol{\Delta}$ : PUA/Novolac/Al $\left(2\right.$ bar, $\left.90^{\circ} \mathrm{C}\right){ }^{3} \boldsymbol{\nabla}: \mathrm{PUA}_{\mathrm{h}} / \mathrm{NPB} / \mathrm{Si}(0.2 \sim 2$ bar, $\left.90^{\circ} \mathrm{C}\right), \diamond: \mathrm{PUA}_{\mathrm{h}} / \mathrm{NPB} / \mathrm{Au}\left(0.2 \sim 2\right.$ bar, $\left.90^{\circ} \mathrm{C}\right), \triangleleft: \mathrm{PUA}_{1} / \mathrm{NPB} / \mathrm{Si}$ $\left(0.2 \sim 2\right.$ bar, $\left.90^{\circ} \mathrm{C}\right)$, and $\triangleright: \mathrm{PUA}_{1} / \mathrm{NPB} / \mathrm{Au}\left(0.2 \sim 2\right.$ bar, $\left.90^{\circ} \mathrm{C}\right)$. An operating window is indicated as the box (filled symbols). Open symbols indicate no detachment.

Calculated values were $W_{\mathrm{PUA} / \mathrm{NPB}}=72.8 \mathrm{~mJ} / \mathrm{m}^{2}, \quad W_{\mathrm{NPB} / \mathrm{Si}}$ $=59.7 \mathrm{~mJ} / \mathrm{m}^{2}$, and $W_{\mathrm{NPB} / \mathrm{Au}}=71.7 \mathrm{~mJ} / \mathrm{m}^{2}$, which satisfy the operation condition of detachment $\left(W_{12}>W_{23}\right)$. The spreading coefficient of a film is given by ${ }^{9}$

$$
S=\gamma_{3}-\gamma_{2}-\gamma_{23},
$$

where $\gamma_{3}$ is the surface energy of substrate, $\gamma_{2}$ is the surface energy of film, and $\gamma_{23}$ is the interfacial energy. If $S$ is positive, the film wets the substrate and no dewetting occurs. If $S$ is negative, the film is thermodynamically unstable, which results in a dewetting at elevated temperatures or at room temperature in the case of a viscous liquid. ${ }^{9} S$ can be obtained from the relation

$$
\gamma_{23}=\gamma_{2}+\gamma_{3}-W_{23} \text {. }
$$

In order to test for four different regimes in Fig. 2(a), various combinations in terms of $S$ and $W_{12} / W_{23}$ were examined by using two PUA molds with different surface tensions $\left(\gamma_{1}=\gamma_{\mathrm{PUA}}=55.8\right.$ or $\left.32.8 \mathrm{~mJ} / \mathrm{m}^{2}\right)$ and two substrates of silicon and gold-coated silicon wafers. Several notable findings are derived from the figure.

First, intrinsic thermodynamic variables govern surface morphologies in detachment nanolithography. As temperature was increased to $90{ }^{\circ} \mathrm{C}\left(T_{g}=96{ }^{\circ} \mathrm{C}\right)$ with the mold in contact with the NPB surface, the mobility of film would be relatively high (not sufficient) to reach thermodynamic equilibrium. Here, type I morphology was found at a positive $S$ and a higher ratio of work of adhesion $\left(W_{12} / W_{23}>1\right)$. NeiDownloaded 15 Jun 2008 to 147.46 .130 .202 Redistribution subject ther detachment nor dewetting was found at a positive $S$ and a lower ratio of work of adhesion $\left(W_{12} / W_{23}<1\right)$ (type II). For relatively lower temperatures $\left(<60{ }^{\circ} \mathrm{C}\right)$, the pattern fidelity was significantly reduced $(<50 \%)$, which suggests the importance of the detachment temperature. ${ }^{4}$

Second, dewetting was observed at a negative $S$ but the resulting pattern was either guided by physical confinement of mold (type III) or consisted of an array of droplets with an apparent periodicity (type IV), a typical pattern for dewetting of a thin liquid film. In type III, the droplets were separated and merged along the line direction. Similar structures were reported when a thin polystyrene film dewetted from a silicon substrate under physical confinement of PDMS walls. ${ }^{10}$ Sometimes, a regular ordering was observed with an apparent wavelength on gold-coated silicon substrate, which is reminiscent of the Rayleigh instability (not shown). In type IV, it appears that the droplets were initially formed along the line direction and then merged rapidly without detachment of a film, resulting in randomly distributed droplets, as shown in Fig. 1(a).

Third, the horizontal demarcation line is clearly dictated by surface tension values that satisfy the operability of detachment lithography $\left(W_{12} / W_{23}>1\right)$ (boundary indicated as the solid line). The vertical demarcation line, however, is not well defined since there would be a range of $S$ in metastable region that does not induce dewetting (boundary indicated as the dotted line). The metastable region is mostly determined by extrinsic conditions of detachment lithography such as temperature and pressure. Hence, the phase diagram in Fig. 2(a) is a snapshot for a given temperature and a pressure, heavily depending on processing parameters used in the experiment.

To elaborate on the effects of process parameters, all of the data reported in literature were collected and plotted in Fig. 2(b) with corresponding values of $S$ and $W_{12} / W_{23}{ }^{11}$ An operating window for detachment nanolithography is indicated as the box (filled symbols); open symbols indicate no detachment. As shown in the figure, detachment nanolithography can be carried out even at a negative $S$ and a high temperature. This situation is understood in that dewetting in metastable region can be suppressed at an elevated temperature for a period of time and the detachment was enhanced by a higher mobility. Nonetheless, $W_{12} / W_{23}$ should be larger than unity for faithful pattern formation regardless of the temperatures used in the experiments.

A few comments are in order about the minimum resolution in detachment nanolithography. As the detachment involves scission of the layer, the difference in the adhesion strength has to be larger than the cohesion strength of the detached layer. As a result, a polymer film is always difficult to detach than an organic layer because of high entanglement among chain molecules. ${ }^{3}$ Therefore, an accurate prediction on the minimum resolution is not an easy task, which would be a complicated function of intrinsic and extrinsic conditions. Here, one example is demonstrated as to how the detachment pressure affects the pattern resolution. Figure 3 shows the patterning images and the corresponding displacement profiles for three pressures applied onto the surface $(0.2,1$, and 2 bars). Displacement profiles over a repeating unit (pitch) of a mold were estimated by applying an indentation problem of a punch, yielding ${ }^{12}$

to AIP license or copyright; see http://apl.aip.org/apl/copyright.jsp 
(a)

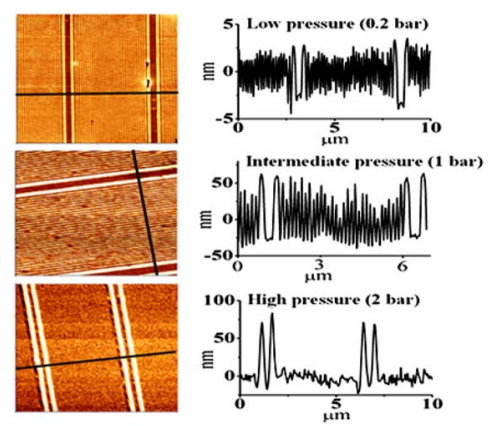

(b)

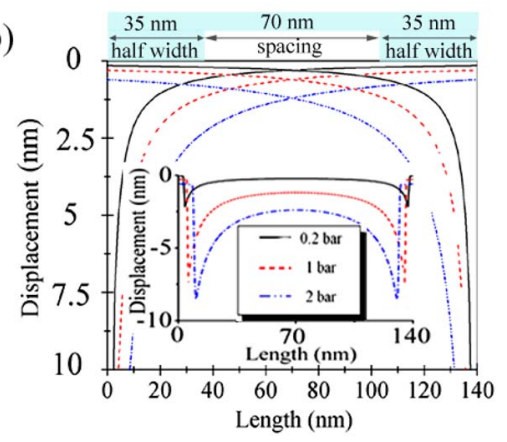

FIG. 3. (Color online) Effects of pressure on detachment nanolithography. (a) Three different nanopatterns and (b) corresponding displacement profiles with different applied pressures $(0.2,1$, and 2 bars) over a repeating unit (pitch) of a mold. At a high pressure of 2 bars, the displacement profiles were overlapped, which results in the detachment of the whole layer containing $70 \mathrm{~nm}$ patterns. The merged displacement profiles are also shown in the inset.

$$
2 \mu u_{z}=\frac{F(1-\nu)}{\pi^{*} r},
$$

where $\mu$ is the shear modulus, $u_{z}$ is the displacement, $F$ is the vertical force, $\nu$ is Poisson's ratio, and $r$ is the distance along the horizontal direction. Young's modulus ( $\sim 2 \mathrm{Gpa})$ and Poisson's ratio $(\sim 0.3)$ of NPB were obtained from literature. ${ }^{13}$ The shear modulus was then calculated from the relation $\mu=E / 2(1+\nu)$. Since the PUA material possesses a fairly high Young's modulus ( $>40 \mathrm{MPa}$ ) compared to rubbery polymers, the collapse of mold's nanopatterns upon physical contact to a substrate could be negligible. ${ }^{14}$

As shown in Fig. 3, a relatively low pressure of 0.2 bar led to an imprinted pattern with a depth of 1-2 nm. At this low pressure, an intimate contact might be achieved but the scission of the layer is not aided by the applied pressure. At an intermediate pressure of $1 \mathrm{bar}$, a detached pattern of high fidelity was obtained, essentially with the same height as that of the original NPB film $(\sim 80 \mathrm{~nm})$. At a high pressure of 2 bars, the whole part of the $70 \mathrm{~nm}$ region $(\sim 5 \mu \mathrm{m}$ wide $)$ was peeled off, leaving behind two $200 \mathrm{~nm}$ lines with $400 \mathrm{~nm}$ spacing at the edge (see Fig. 1 for mold geometry). These results give an insight into the role of pressure for fabricating dense nanoscale patterns. Of particular interest here is the interaction of pressure distributions underneath the detached layer. It is anticipated that the construction interference could give rise to the detachment of the noncontacting part of the layer. This is clearly demonstrated in the displacement profiles in Fig. 3(b) where the two displacement profiles are overlapped, thereby generating a displace- ment to the adjacent noncontacting part. This fact indicates that the interspacing between nanopatterns is also an important parameter that needs to be taken into account.

In summary, we have examined the role of and the interplay between thermodynamic and process parameters in detachment nanolithography. Four different regimes of surface morphology were established depending on the spreading coefficient and the ratio of work of adhesion. For an appropriate range of temperature and pressure, a higher work of adhesion ratio $(>1)$ can ensure faithful detachment even at a negative spreading coefficient, producing well-defined nanopatterns on the surface. Thus, the control of thermodynamic variables is a key element in detachment-based patterning methods. Also, extrinsic conditions such as temperature and pressure play an important role. When the detachment is carried out at an elevated temperature in a metastable state, the detachment is facilitated by a high mobility of the film without generating droplets. The effects of pressure are also deeply related to faithful detachment and the minimum resolution of the resulting pattern.

This work was supported by the Micro Thermal System Research Center of Seoul National University and in part by the Ministry of Science and Technology through the Nanoscopia Center of Excellence.

${ }^{1}$ K. J. Lee, M. J. Motala, M. A. Meitl, W. R. Childs, E. Menard, A. K. Shim, J. A. Rogers, and R. G. Nuzzo, Adv. Mater. (Weinheim, Ger.) 17, 2332 (2005); J. Zaumseil, M. A. Meitl, J. W. P. Hsu, B. R. Acharya, K. W. Baldwin, Y. L. Loo, and J. A. Rogers, Nano Lett. 3, 1223 (2003); Y. L. Loo, T. Someya, K. W. Baldwin, Z. N. Bao, P. Ho, A. Dodabalapur, H. E. Katz, and J. A. Rogers, Proc. Natl. Acad. Sci. U.S.A. 99, 10252 (2002). ${ }^{2}$ C. Kim, P. E. Burrows, and S. R. Forrest, Science 288, 831 (2000).

${ }^{3}$ S. M. Seo, J. Y. Park, and H. H. Lee, Appl. Phys. Lett. 86, 133114 (2005). ${ }^{4}$ Z. Wang, J. Zhang, R. Xing, J. F. Yuan, D. H. Yan, and Y. C. Han, J. Am. Chem. Soc. 125, 15278 (2003).

${ }^{5}$ J. H. Choi, D. Kim, P. J. Yoo, and H. H. Lee, Adv. Mater. (Weinheim, Ger.) 17, 166 (2005).

${ }^{6}$ S. J. Choi, P. J. Yoo, S. J. Baek, T. W. Kim, and H. H. Lee, J. Am. Chem. Soc. 126, 7744 (2004).

${ }^{7}$ M. A. Meitl, Z. T. Zhu, V. Kumar, K. J. Lee, X. Feng, Y. Y. Huang, I. Adesida, R. G. Nuzzo, and J. A. Rogers, Nat. Mater. 5, 33 (2006).

${ }^{8}$ J. K. Kim, J. W. Park, H. Yang, M. Choi, J. H. Choi, and K. Y. Suh, Nanotechnology 17, 940 (2006).

${ }^{9}$ Souheng Wu, Polymer Interface and Adhesion (Dekker, New York, 1982), p. 10.

${ }^{10}$ K. Y. Suh, J. Park, and H. H. Lee, J. Chem. Phys. 116, 7714 (2002).

${ }^{11}$ The calculated surface energies from the literature are as follows: $\gamma_{\mathrm{Alq} 3}$ $=36.4 \mathrm{~mJ} / \mathrm{m}^{2}, \quad \gamma_{\text {novolac }}=44.2 \mathrm{~mJ} / \mathrm{m}^{2}, \quad \gamma_{\mathrm{NPB}}=31.8 \mathrm{~mJ} / \mathrm{m}^{2}, \quad \gamma_{\text {ITO }}$ $=32.4 \mathrm{~mJ} / \mathrm{m}^{2}, \gamma_{\mathrm{Al}}=44.9 \mathrm{~mJ} / \mathrm{m}^{2}, \gamma_{\mathrm{Si}}=63.2 \mathrm{~mJ} / \mathrm{m}^{2}$, and $\gamma_{\mathrm{Au}}=43.9 \mathrm{~mJ} / \mathrm{m}^{2}$. Also, work of adhesion values are $W_{\text {epoxy } / \text { Alq } 3}=64.5 \mathrm{~mJ} / \mathrm{m}^{2}, W_{\text {Alq } 3 / \text { TTO }}$ $=41.0 \mathrm{~mJ} / \mathrm{m}^{2}, \quad W_{\text {PDMS } / \text { Alq } 3}=57.6 \mathrm{~mJ} / \mathrm{m}^{2}, W_{\text {PUA } / \text { novolac }}=81.7 \mathrm{~mJ} / \mathrm{m}^{2}$, and $W_{\text {novolac } / \mathrm{Al}}=78.2 \mathrm{~mJ} / \mathrm{m}^{2}$. According to our experiment results, the values are $W_{\mathrm{PUA}(\text { high }) / \mathrm{NPB}}=72.8 \mathrm{~mJ} / \mathrm{m}^{2}, \quad W_{\mathrm{PUA}(\text { low }) / \mathrm{NPB}}=39.3 \mathrm{~mJ} / \mathrm{m}^{2}, \quad W_{\mathrm{NPB} / \mathrm{Si}}$ $=59.7 \mathrm{~mJ} / \mathrm{m}^{2}$, and $W_{\mathrm{NPB} / \mathrm{Au}}=71.7 \mathrm{~mJ} / \mathrm{m}^{2}$. The calculated interface energies are as follows: $\gamma_{\mathrm{Alq} 3 / \mathrm{ITO}}=27.8 \mathrm{~mJ} / \mathrm{m}^{2}, \gamma_{\text {novolac } / \mathrm{Al}}=10.9 \mathrm{~mJ} / \mathrm{m}^{2}$, and $\gamma_{\mathrm{NPB} / \mathrm{Si}}=35.3 \mathrm{~mJ} / \mathrm{m}^{2}$, and $\gamma_{\mathrm{NPB} / \mathrm{Si}}=4.0 \mathrm{~mJ} / \mathrm{Au}^{2}$. The spreading coefficients are: $-31.8 \mathrm{~mJ} / \mathrm{m}^{2}$ [Alq3 on indium tin oxide (ITO) coated glass], $-10.2 \mathrm{~mJ} / \mathrm{m}^{2}$ (novolac on aluminum coated glass), $-3.9 \mathrm{~mJ} / \mathrm{m}^{2}$ (NPB on silicon substrate), and $+8.1 \mathrm{~mJ} / \mathrm{m}^{2}$. (NPB on gold coated silicon substrate).

${ }^{12}$ J. R. Barber, Elasticity (Kluwer, Dordrecht, Boston, 1992), p. 223.

${ }^{13}$ C. Kim, Y. Cao, W. O. Soboyejo, and S. R. Forrest, J. Appl. Phys. 97, $113512(2005)$

${ }^{14}$ Y. G. Y. Huang, W. X. Zhou, K. J. Hsia, E. Menard, J. U. Park, J. A. Rogers, and A. G. Alleyne, Langmuir 21, 8058 (2005). 